\title{
Micoses sistêmicas: fatores associados ao óbito em pacientes com infecção pelo vírus da imunodeficiência humana, Cuiabá, Estado de Mato Grosso, 2005-2008
}

\author{
Systemic mycosis: factors associated with death among patients infected with the \\ human immunodeficiency virus, Cuiabá, State of Mato Grosso, Brazil, 2005-2008
}

\author{
Luciano Correa Ribeiro ${ }^{1}$, Rosane Christine Hahn ${ }^{1,2,3}$, Olivia Cometi Favalessa ${ }^{1}$, \\ Tomoko Tadano ${ }^{1}$ e Cor Jesus Fernandes Fontes ${ }^{1,2}$
}

\begin{abstract}
RESUMO
A prevalência de micose sistêmica entre 1.300 pacientes portadores de HIV/Aids de Cuiabá, Mato Grosso foi de 4,6\%, no período de 2005-2008. As espécies de fungos isoladas foram o Cryptococcus neoformans (50\%), Cryptococcus gattii (1,6\%), Cryptococcus spp (6,6\%), Histoplasma capsulatum (38,3\%) e Paracoccidioides brasiliensis (3,3\%). Óbito foi registrado em 32 (53,3\%) pacientes, sendo a criptococose a principal causa. A contagem de linfócitos T CD4+ foi baixa e semelhante entre os pacientes que sobreviveram ou faleceram por micose sistêmica. 0 etilismo (0R:8,2; IC95\%: 1,4-62,1; p=0,005) e o nível médio de desidrogenase lática [758 (182) U/L vs 416 (268) U/L; p<0,001] foram as características independentemente associadas ao óbito dos pacientes do estudo. Os resultados mostram alta letalidade por micoses sistêmicas em pacientes portadores de HIV/Aids de Cuiabá e sugerem que características clínico-laboratoriais tais como o etilismo e a elevação precoce da desidrogenase lática podem ser fatores relacionados ao pior prognóstico nessas condições.
\end{abstract}

Palavras-chaves: Vírus da imunodeficiência humana. Micoses sistêmicas. Letalidade.

\begin{abstract}
Between 2005 and 2008, the prevalence of systemic mycosis among 1,300 HIV/AIDS patients in Cuiabá, Mato Grosso, was 4.6\%. The fungus species isolated were Cryptococcus neoformans in 50\%, Cryptococcus gattii in 1.6\%, Cryptococcus spp in 6.6\%, Histoplasma capsulatum in $38.3 \%$ and Paracoccidioides brasiliensis in 3.3\%. Death was recorded in the cases of 32 patients (53.3\%), and cryptococcosis was the main cause. The CD4+ T lymphocyte count was low and similar among patients who survived or died due to systemic mycosis. The factors independently associated with the deaths of these patients were alcoholism (OR: 8.2; 95\% CI: 1.4-62.1; p = 0005) and the mean level of lactate dehydrogenase [758 (182) U/1 vs. 416 (268) U/; p $<0001$ ]. The findings showed that systemic mycosis was highly lethal among the patients with HIV/AIDS in Cuiabá and suggested that clinical-laboratory characteristics such as alcoholism and early elevation of lactate dehydrogenase may be factors relating to worse prognosis under these conditions.
\end{abstract}

Key-words: Human immunodeficiency virus. Systemic mycosis. Lethality.

Mesmo com a queda progressiva da incidência de infecções oportunistas, após o advento da moderna terapia antirretroviral potente ou high activity antiretroviral therapy (HAART) ${ }^{17}$, estas complicações ainda constituem a principal causa de morbidade e mortalidade para a população portadora do vírus da imunodeficiência humana (Aids) resultando muitas vezes em hospitalização, e requerendo, em algumas situações, tratamentos

1. Hospital Universitário Júlio Müller, Universidade Federal do Mato Grosso, Cuiabá, MT, 2. Associação Mato-Grossense Para Estudo das Endemias Tropicais, Cuiabá, MT, 3. Hospital Geral Universitário, Universidade de Cuiabá, Cuiabá, MT.

Apoio financeiro: Este estudo foi financiado pelo Programa Nacional de Aids, através da UNESCO.

Endereço para correspondência: Dr. Luciano Corrêa Ribeiro. Rua Modena 31, Bairro Jardim Itália, 78060-808 Cuiabá,MT.

e-mail: lucorrea@terra.com.br

Recebido para publicação em 17/12/2009

Aceito em 27/11/2009 dispendiosos e muito tóxicos, podendo resultar em encurtamento da sobrevida destes pacientes 5 .

Apesar da tuberculose representar ainda a principal complicação oportunista, com crescimento exponencial significativo, também, é crescente a incidência de infecção fúngica invasiva em determinadas populações, como no sudoeste asiático e outros países em desenvolvimento, em decorrência do elevado grau de imunossupressão ${ }^{636}$. Shen e cols ${ }^{37}$ relataram que os pacientes portadores do HIV podem apresentar uma variedade de infecções fúngicas no decorrer de sua trajetória, com aumento significativo do número de casos, ocasionando uma elevada letalidade, já que o diagnóstico precoce é difícil devido sintomatologia clínica pouco específica.

0 objetivo deste estudo foi identificar fatores associados ao óbito causado por micoses sistêmicas em pacientes com HIV/Aids que receberam tratamento nas unidades especializadas para assistência ao portador de HIV/Aids de Cuiabá, Mato Grosso, 2005-2008. 


\section{PACIENTES E MÉTODOS}

Estudo epidemiológico de corte transversal, cujas informações foram obtidas da análise de prontuários de pacientes acompanhados nas unidades especializadas para assistência ao portador de HIV/ Aids, de Cuiabá, Mato Grosso, 2005-2008. Foram levantados os dados dos prontuários que continham as informações sobre 0 acompanhamento clínico do paciente, tanto em sua fase hospitalar quanto ambulatorial de assistência, nos seguintes hospitais: Hospital Pronto Socorro Municipal de Cuiabá, Santa Casa de Misericórdia de Cuiabá, Hospital Geral Universitário e Hospital Universitário Júlio Muller.

Para identificar fatores associados ao óbito dos pacientes com micose sistêmica, associada ao HIV/Aids, foram priorizados os seguintes dados clínicos: principais sinais e sintomas, registro ou evidência, no prontuário, de infecção fúngica prévia ou de uso prévio de antifúngicos após o diagnóstico de Aids e presença de outras infecções oportunistas associadas. Informações epidemiológicas e demográficas tais como escolaridade, idade, sexo e hábito de usar fumo e álcool também foram registradas. Foram classificados como etilistas e tabagistas os pacientes cujos registros de prontuários constavam qualquer menção a esses hábitos.

$\mathrm{Na}$ avaliação laboratorial, foram coletados dados hematológicos, bioquímicos, contagem de linfócitos CD4+ e carga viral, no momento da internação. As seguintes variáveis categóricas relacionadas aos resultados observados nos exames laboratoriais foram definidas: anemia, se a concentração de hemoglobina foi inferior a 10g/dL; insuficiência renal, se o nível sérico de uréia foi superior a $50 \mathrm{mg} / \mathrm{dL}$; hipoalbuminemia, se a albumina sérica foi inferior a 3g/dL; enzimas hepáticas elevadas, se $o$ valor da aspartato-aminotransferase (AST) ou alaninaaminotransferase (ALT) foi superior a 40U/L; desidrogenase lática elevada, se a DHL foi superior a 400U/L. A carga viral do HIV foi avaliada pelo logaritmo (base 10) do número de cópias de DNA constatado.

0 diagnóstico da infecção fúngica de todos pacientes do estudo foi feito no laboratório de microbiologia da instituição onde esteve hospitalizado. A discriminação específica dos microorganismos de interesse no estudo foi realizada no Laboratório de Micologia da Faculdade de Ciências Médicas/ Universidade Federal do Mato Grosso. As técnicas utilizadas para essa identificação foram o exame micológico direto, a coloração pela tinta Nanquim, para identificação de Cryptococcus sp, cultura com meios de Sabouraud dextrose, Mycosel ou Fava-Netto e a realização de provas específicas, objetivando a distinção entre as espécies Cryptococcus neoformans e Cryptococcus gattii. Para o presente estudo, classificou-se como portador de micose sistêmica todo paciente cujo diagnóstico etiológico de sua infecção oportunista foi concluído como criptococose, histoplasmose e paracoccidioidomicose.

Para avaliar a força de associação entre variáveis categóricas associadas ao óbito, foram determinados a razão de chances (odds ratio) e respectivo intervalo de confiança (IC95\%). Diferenças observadas entre proporções foram analisadas pelo teste de qui-quadrado com correção de Yates ou pelo teste de Fisher. Para análise das variáveis contínuas, associadas ao óbito, valeu-se da comparação da média e desvio padrão de cada grupo, utilizando-se o teste $t$ de Student. Para todas essas análises, foi considerado o nível de significância de 95\%. Este estudo foi aprovado pelo Comitê de Ética em Pesquisa (CEP) do Hospital Universitário Júlio Muller (HUJM), pelo Parecer no 226/CEP-HUJM/2005.

Todas as variáveis que mostraram associação em análise univariada, com probabilidade de erro alfa inferior 0,1 , foram posteriormente incluídas em modelo de regressão logística multivariada, para determinar os fatores independentemente associados ao óbito, utilizando o programa Stata, versão 8.0.

\section{RESULTADOS}

Foram avaliados os prontuários de 1.300 pacientes inscritos nas unidades especializadas para assistência ao portador de HIV/ Aids de Cuiabá, Mato Grosso, no período de 2005-2008, sendo possível identificar infecção fúngica (de qualquer natureza) em 175 (13,5\%; IC95\%: 11,7\%-15,5\%) pacientes. Micose sistêmica foi constatada em 60 (34,3\%; IC95\%: 27,4\%-41,9\%) desses pacientes, sendo 37 (61,7\%) do sexo masculino e $23(38,3 \%)$ do sexo feminino, numa razão masculino: feminino de 1,6:1. A idade desses pacientes variou de 11 a 59 anos, com média (DP) de $36,5(9,7)$ anos. Destacaram-se pacientes com mais de 30 dias de início de sintomas $(67,2 \%)$, baixo nível de escolaridade $(85,6 \%)$, referindo uso frequente de bebidas alcoólicas $(67,3 \%)$ e hábito de fumar cigarros (74,5\%). Infecção fúngica pregressa e uso anterior de medicamentos com ação antifúngica foram encontrados nos prontuários de $17(30,4 \%)$ e $16(28,6 \%)$ pacientes, respectivamente (Tabela 1). Dados clínicos, de interesse para o estudo, foram anotados de 56 prontuários dos 60 pacientes com micose sistêmica. Os principais sinais e sintomas referidos por esses pacientes, quando do diagnóstico da micose sistêmica foram, em geral, inespecíficos. Predominaram os achados de fraqueza muscular, febre, palidez cutânea, emagrecimento, adenomegalia, cefaléia e confusão mental.

As alterações laboratoriais encontradas por ocasião do diagnóstico da micose sistêmica confirmaram anemia, plaquetopenia e hipoalbuminemia, atestadas pelos achados (média \pm DP) de hemoglobina de $9,3 \pm 2,6 \mathrm{~g} / \mathrm{dL}$, hematócrito de $27,5 \% \pm 7,1 \%$, contagem de plaquetas de $170.145 \pm 117.602 / \mathrm{mm}^{3}$ e albumina sérica de $2,8 \pm 0,7 \mathrm{~g} / \mathrm{dL}$. Os níveis séricos médios (DP) das enzimas aspartato aminotransferase, alanina aminotransferase, desidrogenase láctica e fosfatase alcalina foram $101,7(122,3)$ U/L, 50,4 (39,4) U/L, 560,3 $(287,9) \mathrm{U} / \mathrm{L}$ e $232,5(218,1) \mathrm{U} / \mathrm{L}$, respectivamente. Evidência importante de imunodepressão foi constatada pelo baixo nível de linfócitos T CD4 (media \pm DP de $82,3 \pm 85,2$ células) e alta carga viral (média \pm DP de $1.054 .016 \pm 1.787 .576$ cópias/mL) (Tabela 2).

A positividade da investigação micológica dos 60 pacientes do estudo foi demonstrada em espécimes clínicos de 56 (93,3\%) exames micológicos diretos, 53 (88,3\%) culturas e 7 (11,6\%) exames anatomopatológicos. Em vários deles, a micose 
foi confirmada em mais de um sítio anatômico. Os principais sítios anatômicos onde foram isolados os fungos causadores da micose sistêmica diagnosticada nesses pacientes foram líquido cefalorraquidiano $(56,7 \%)$, medula óssea $(30 \%)$, pulmão

\section{TABELA 1}

Características clínico-demográficos dos pacientes portadores de HIV e co-infectados por fungos causadores de micose sistêmica, em acompanhamento nas unidades especializadas para assistência ao portador de HIV/Aids, Mato Grosso, 2005-2008.

\begin{tabular}{|c|c|c|}
\hline Característica & Número & Percentagem \\
\hline Idade em anos (média \pm DP) & 36,5 & $(9,7)$ \\
\hline \multicolumn{3}{|l|}{ Tempo de sintomas em dias $\left(\mathrm{n}^{\circ}=57\right)$} \\
\hline$\leq 30$ & 18 & 32,7 \\
\hline$>30$ & 37 & 67,2 \\
\hline \multicolumn{3}{|l|}{ Sexo } \\
\hline masculino & 37 & 61,7 \\
\hline feminino & 23 & 38,3 \\
\hline Escolaridade $\left(\mathrm{n}^{0}=56\right)$ & 24 & 42,8 \\
\hline nenhuma & 24 & 42,8 \\
\hline fundamental & 24 & 42,8 \\
\hline médio ou superior & 8 & 14,4 \\
\hline Etilismo $\left(\mathrm{n}^{\mathrm{0}}=55\right)$ & 37 & 67,3 \\
\hline Tabagismo $\left(\mathrm{n}^{0}=55\right)$ & 41 & 74,5 \\
\hline Infecção fúngica prévia $\left(\mathrm{n}^{\circ}=56\right)$ & 17 & 30,4 \\
\hline Uso prévio de antifúngico $\left(\mathrm{n}^{0}=56\right)$ & 16 & 28,6 \\
\hline Uso prévio de TARV-P $\left(\mathrm{n}^{0}=52\right)$ & 22 & 42,3 \\
\hline Pneumocistose associada $\left(\mathrm{n}^{0}=57\right)$ & 17 & 29,8 \\
\hline Tuberculose associada $\left(\mathrm{n}^{0}=57\right)$ & 15 & 26,3 \\
\hline Neurotoxoplasmose associada $\left(\mathrm{n}^{0}=57\right)$ & 8 & 14,0 \\
\hline
\end{tabular}

HIV: vírus da imunodeficiência humana, Aids: síndrome da imunodeficiência adquirida, DP: desvio padrão, TARV-P: terapia antirretroviral potente. Observação: variação do $\mathrm{n}^{0}$ deveu-se à falta de informações da variável no prontuário do paciente.

\section{TABELA 2}

Características laboratoriais dos 60 pacientes portadores de HIV e micose sistêmica, em acompanhamento nas unidades especializadas para assistência ao portador de HIV/Aids, Mato Grosso, 2005-2008.

\begin{tabular}{lcr}
\hline Característica & Média & Desvio padrão \\
\hline Hemoglobina (g/dL) & 9,3 & 2,6 \\
Hematócrito (\%) & 27,5 & 7,1 \\
Leucócitos/mm & 4.902 & 3.800 \\
Linfócitos/mm & 800 & 831 \\
Plaquetas/mm ${ }^{3}$ & 170.145 & 117.602 \\
Albumina (g/dL) & 2,8 & 0,7 \\
Uréia (g/dL) & 46,6 & 31,4 \\
Creatinina (g/dL) & 1,2 & 0,6 \\
AST (U/L) & 101,7 & 122,3 \\
ALT (U/L) & 50,4 & 39,4 \\
DHL (U/L) & 560,3 & 287,9 \\
Fosfatase alcalina (U/L) & 232,5 & 218,1 \\
Potássio sérico (mEq/L) & 3,76 & 0,8 \\
VHS 1 ${ }^{a}$ hora (mm) & 66,2 & 41,0 \\
Linfócitos T CD4+ (células/mm³) & 82,3 & 85,2 \\
Carga viral (cópias/mL) & 1.054 .016 & 1.787 .576 \\
Raio-X de tórax alterado & 33 (n\%) & $55,0(\%)$ \\
\hline HIV: vírus da imunodeficiência humana, Aids: síndrome da imunodeficiência \\
adquirida, AST: aspartato-aminotransferase, ALT: alanina-aminotransferase, \\
DHL: desidrogenase lática, VHS: velocidade de hemossedimentação. &
\end{tabular}

$(13,3 \%)$ e boca (11,7\%). A hemocultura e a urinocultura foram úteis para a confirmação da infecção fúngica em $8(13,3 \%)$ e $4(6,7 \%)$ dos 53 pacientes com cultura positiva, respectivamente. Dos fungos isolados no sangue, dois foram agentes causadores de histoplasmose e seis de criptococose. As espécies de fungos isoladas nos 60 pacientes do estudo estão apresentadas na Tabela 3.

Evolução para alta ou óbito foi registrada em 24 (40\%) e $32(53,3 \%)$ pacientes, respectivamente. De $4(6,7 \%)$ pacientes, não foi possível levantar observação quando ao desfecho evolutivo da micose sistêmica. Entre 37 pacientes, que tinham informação sobre etilismo referida no prontuário, $20(54,1 \%)$ evoluíram para óbito durante o tratamento da micose sistêmica. Entre 16 pacientes que não referiram uso de bebidas alcoólicas, apenas $2(12,5 \%)$ foram a óbito. A probabilidade de etilismo foi maior e estatisticamente significante, entre os pacientes que evoluíram para óbito (OR:8,2; IC95\%: 1,4-62,1; p=0,005). Outras infecções concomitantes, tais como tuberculose $(\mathrm{p}=0,47)$, pneumocistose $(\mathrm{p}=0,08)$ e neurotoxoplasmose $(\mathrm{p}=0,78)$, não se associaram à evolução letal desses pacientes. Da mesma forma, as demais variáveis clínicas e demográficas levantadas dos pacientes também não apresentaram associação com o óbito causado por micose sistêmica, nesses pacientes (Tabela 4).

Algumas características laboratoriais destacaram-se entre os pacientes que evoluíram para o óbito. Nível médio (DP) de albumina sérica foi menor e estatisticamente significante,

TABELA 3

Principais agentes fúngicos isolados dos 60 pacientes portadores de HIV/Aids e micose sistêmica, em acompanhamento nas unidades especializadas para assistência ao portador de HIV/Aids, Mato Grosso, 2005-2008.

\begin{tabular}{|c|c|c|c|}
\hline Agente & $\begin{array}{c}\text { Principais sítios } \\
\text { de isolamento }\end{array}$ & Número & Percentagem \\
\hline \multirow[t]{6}{*}{ Cryptococcus neoformans $\left(\mathrm{n}^{0}=30\right)$} & liquor & 29 & 96,7 \\
\hline & pulmão & 4 & 13,3 \\
\hline & corrente sanguínea & 3 & 10,0 \\
\hline & urina & 3 & 10,0 \\
\hline & linfonodo & 2 & 6,7 \\
\hline & medula óssea & 1 & 3,3 \\
\hline \multirow[t]{3}{*}{ Cryptococcus gattii $\left(\mathrm{n}^{0}=1\right)$} & líquor & 1 & 100,0 \\
\hline & pele & 1 & 100,0 \\
\hline & corrente sanguínea & 1 & 100,0 \\
\hline \multirow[t]{2}{*}{ Cryptococcus $\operatorname{spp}\left(\mathrm{n}^{0}=4\right)$} & liquor & 3 & 75,0 \\
\hline & corrente sanguínea & 2 & 66,7 \\
\hline \multirow[t]{9}{*}{ Histoplasma capsulatum $\left(\mathrm{n}^{\circ}=23\right)$} & medula óssea & 17 & 73,9 \\
\hline & boca & 5 & 21,7 \\
\hline & corrente sanguínea & 2 & 8,7 \\
\hline & pulmão & 2 & 8,7 \\
\hline & linfonodos & 1 & 4,3 \\
\hline & corrente sanguínea & 1 & 4,3 \\
\hline & liquor & 1 & 4,3 \\
\hline & intestino & 1 & 4,3 \\
\hline & urina & 1 & 4,3 \\
\hline Paracoccidiodes brasiliensis $\left(\mathrm{n}^{0}=2\right)$ & pulmão & 2 & 100,0 \\
\hline
\end{tabular}

HIV: vírus da imunodeficiência humana, Aids: síndrome da imunodeficiência adquirida. Obs: alguns pacientes tiveram o agente fúngico isolado de vários sítios. 
TABELA 4

Fatores clínicos e demográficos associados ao óbito dos pacientes portadores de HIV e co-infectados por fungos causadores de micose sistêmica, em acompanhamento nas unidades especializadas para assistência ao portador de HIV/Aids, Mato Grosso, 2005-2008.

\begin{tabular}{|c|c|c|c|c|c|c|c|}
\hline \multirow[b]{3}{*}{ Fatores } & \multicolumn{4}{|c|}{ Óbito } & \multirow[b]{3}{*}{ OR } & \multirow[b]{3}{*}{ IC95\% } & \multirow[b]{3}{*}{$\mathrm{p}$} \\
\hline & \multicolumn{2}{|c|}{$\operatorname{sim}$} & \multicolumn{2}{|c|}{ não } & & & \\
\hline & $n^{0}$ & $\%$ & $n^{0}$ & $\%$ & & & \\
\hline \multicolumn{8}{|l|}{ Sexo } \\
\hline masculino & 15 & 42,9 & 20 & 57,1 & 1,00 & $0,29-3,47$ & 1,00 \\
\hline feminino & 9 & 42,9 & 12 & 57,1 & & & \\
\hline \multicolumn{8}{|l|}{ Escolaridade } \\
\hline nenhuma & 12 & 50,0 & 12 & 50,0 & 2,00 & $0,66-6,03$ & 0,83 \\
\hline fundamental & 7 & 31,8 & 15 & 68,2 & 0,53 & $0,17-1,64$ & 0,86 \\
\hline média & 3 & 37,5 & 5 & 62,5 & 0,85 & $0,18-4,01$ & 0,84 \\
\hline \multicolumn{8}{|l|}{ Etilismo } \\
\hline $\operatorname{sim}$ & 20 & 54,1 & 17 & 45,9 & 8,24 & $1,42-62,1$ & 0,005 \\
\hline não & 2 & 12,5 & 14 & 87,5 & & & \\
\hline \multicolumn{8}{|l|}{ Tabagismo } \\
\hline $\operatorname{sim}$ & 18 & 45,0 & 22 & 55,0 & 1,84 & $0,71-8,73$ & 0,37 \\
\hline não & 4 & 30,8 & 9 & 69,2 & & & \\
\hline \multicolumn{8}{|c|}{ Infecção fúngica prévia } \\
\hline $\operatorname{sim}$ & 7 & 41,2 & 10 & 58,8 & 1,03 & $0,27-3,88$ & 0,97 \\
\hline não & 15 & 40,5 & 22 & 59,5 & & & \\
\hline \multicolumn{8}{|c|}{ Uso prévio de antifúngico } \\
\hline $\operatorname{sim}$ & 7 & 3,8 & 9 & 56,3 & 1,19 & $0,31-4,60$ & 0,77 \\
\hline não & 15 & 39,5 & 23 & 60,5 & & & \\
\hline \multicolumn{8}{|c|}{ Tuberculose associada } \\
\hline $\operatorname{sim}$ & 7 & 50,0 & 7 & 50,0 & 1,56 & $0,39-6,34$ & 0,47 \\
\hline não & 16 & 39,0 & 25 & 61,0 & & & \\
\hline \multicolumn{8}{|c|}{ Neurotoxoplasmose associada } \\
\hline $\operatorname{sim}$ & 3 & 37,5 & 5 & 62,5 & 0,81 & $0,13-4,66$ & 0,78 \\
\hline não & 20 & 42,6 & 27 & 57,4 & & & \\
\hline \multicolumn{8}{|c|}{ Pneumocistose associada } \\
\hline $\operatorname{sim}$ & 10 & 58,8 & 7 & 41,2 & 2,74 & $0,73-10,68$ & 0,08 \\
\hline não & 13 & 34,2 & 25 & 65,8 & & & \\
\hline \multicolumn{8}{|l|}{ Uso prévio de TARV-P } \\
\hline $\operatorname{sim}$ & 9 & 42,9 & 12 & 57,1 & 0,94 & $0,26-3,43$ & 0,91 \\
\hline não & 12 & 41,4 & 17 & 58,6 & & & \\
\hline \multicolumn{8}{|l|}{ Dias de sintomas } \\
\hline $0-30$ & 6 & 33,3 & 12 & 66,7 & 0,67 & $0,17-2,53$ & 0,50 \\
\hline$>30$ & 15 & 42,9 & 20 & 57,1 & & & \\
\hline Idade (média $\pm D P$ ) & \multicolumn{2}{|c|}{$36,3 \pm 7,8$} & \multicolumn{2}{|c|}{$36,0 \pm 11,8$} & 1,00 & $0,95-1,06$ & 0,91 \\
\hline
\end{tabular}

IC95\%: intervalo de confiança de 95\%, HIV: vírus da imunodeficiência humana adquirida, Aids: síndrome da imunodeficiência humana adquirida, TARV-P: terapia antirretroviral potente, DP: desvio padrão.Obs: variação do n⿳0 ${ }^{0}$ deveu-se à falta de informações da variável no prontuário do paciente.

entre os pacientes que faleceram, quando comparado com os sobreviventes [2,5 $(0,6) \mathrm{g} / \mathrm{dL}$ vs 3,1 $(0,6) \mathrm{g} / \mathrm{dL} ; \mathrm{p}<0,001]$. A concentração média (DP) da uréia foi de $60,1(42,1) \mathrm{mg} / \mathrm{dL}$ no soro dos pacientes que faleceram e de $36,8(15,5) \mathrm{mg} / \mathrm{dL}$ $(\mathrm{p}=0,006)$. Maior nível médio de DHL foi observado no grupo dos pacientes que evoluíram para o óbito [758 (182) U/L vs 416 (268) U/L; p <0,001]. Embora a proporção de isolamento de fungos em hemocultura tenha sido maior (5/32 $\times 3 / 24)$, entre os pacientes que evoluíram para óbito, a diferença observada não foi estatisticamente significante (Tabela 5).

Os dois parâmetros laboratoriais que são indicadores da agressão ao sistema imune pelo HIV, isto é, a carga viral e a contagem de linfócitos T $\mathrm{CD} 4^{+}$mostraram comportamento de doença avançada, tanto no grupo sobrevivente, quanto no grupo que evoluiu para o óbito. No entanto, entre os pacientes que faleceram, a carga viral do HIV (média: 12,4 log; DP: 3,6 log cópias/mL) foi maior $(\mathrm{p}=0,01)$, quando comparada aos valores observados entre os sobreviventes (média: 9,3 log; DP: 3,7 log cópias/mL). Para a contagem dos linfócitos T CD4+, a diferença observada entre os grupos não foi estatisticamente significante $(\mathrm{p}=0,71)$. Os demais parâmetros laboratoriais avaliados, não apresentaram associação com a evolução da micose sistêmica para o óbito (Tabela 5).

Para avaliar possível efeito de confundimento na associação observada, entre a evolução letal da micose e relato de etilismo, 
TABELA 5

Fatores laboratoriais associados ao óbito de pacientes portadores de HIV e fungos causadores de infecção sistêmica, em acompanhamento nas unidades especializadas para assistência ao portador de HIV/Aids, Mato Grosso, 2005-2008.

\begin{tabular}{|c|c|c|c|c|c|}
\hline \multirow[b]{3}{*}{ Fatores (média $\pm \mathrm{DP}$ ) } & \multicolumn{4}{|c|}{ Óbito } & \multirow[b]{3}{*}{$\mathrm{p}$} \\
\hline & \multicolumn{2}{|c|}{$\operatorname{sim}$} & \multicolumn{2}{|c|}{ não } & \\
\hline & média & DP & média & DP & \\
\hline Hemoglobina em g/dL & 9,9 & 2,7 & 9,9 & 2,6 & 0,06 \\
\hline Hematócrito (\%) & 25,8 & 7,1 & 28,7 & 7,1 & 0,15 \\
\hline Leucócitos/mm³ & 5.756 & 5.141 & 4.917 & 4.374 & 0,52 \\
\hline Linfócitos/mm $\mathrm{mm}^{3}$ & 889 & 1.088 & 947 & 1.314 & 0,86 \\
\hline Linfóctios T CD4+/mm 3 & 90 & 98 & 82 & 80 & 0,71 \\
\hline Plaquetas/ $\mathrm{mm}^{3}$ & 149.957 & 117.101 & 193.378 & 114.559 & 0,13 \\
\hline Albumina $(\mathrm{g} / \mathrm{dL})$ & 2,5 & 0,59 & 3,1 & 0,62 & $<0,001$ \\
\hline Uréia (g/dL) & 60,1 & 42,1 & 36,8 & 15,5 & 0,006 \\
\hline AST $(g / d L)$ & 138,9 & 165,9 & 77 & 69,0 & 0,07 \\
\hline $\operatorname{ALT}(\mathrm{g} / \mathrm{dL})$ & 58,2 & 48,9 & 46,0 & 30,2 & 0,26 \\
\hline Relação AST/ALT & 1,9 & 1,4 & 2,3 & 1,6 & 0,34 \\
\hline Creatinina (g/dL) & 1,4 & 0,8 & 1,2 & 0,4 & 0,11 \\
\hline Glicose (g/dL) & 93 & 22 & 98 & 18 & 0,40 \\
\hline Desidrogenase lática (U/L) & 758 & 182 & 416 & 268 & $<0,001$ \\
\hline Potássio sérico $(\mathrm{mEq} / \mathrm{L})$ & 3,9 & 0,9 & 3,8 & 0,7 & 0,64 \\
\hline Carga viral (cópias/mL) Log & 12,4 & 3,6 & 9,3 & 3,7 & 0,01 \\
\hline Fosfatase alcalina & 280 & 247 & 198 & 189 & 0,19 \\
\hline VHS (primeira hora) & 71 & 41 & 63 & 48 & 0,50 \\
\hline
\end{tabular}

AST: aspartato-aminotransferase, ALT: alanina-aminotransferase, DP: desvio padrão, VHS: velocidade de hemossedimentação.Obs: variação do nº deveu-se à falta de informações da variável no prontuário do paciente.

concomitância de pneumocistose, na ocasião do diagnóstico da micose sistêmica, anemia, hipoalbuminemia, insuficiência renal, elevação da DHL sérica e elevação das enzimas hepáticas, um modelo de regressão logística multivariada foi construído, incluindo-se manualmente as variáveis que apresentaram associação na análise univariada. Em virtude do baixo número de pacientes com informação disponível no prontuário, sobre a carga viral de HIV no momento da admissão, essa variável não pode ser incluída no modelo. Além das variáveis descritas neste parágrafo, incluíram-se também no modelo a idade e a contagem de linfócitos T $\mathrm{CD}^{+}$dos pacientes, em virtude de naturalmente estarem relacionadas à pior evolução clínica. Permaneceram independentemente associadas ao óbito apenas o relato de etilismo e a DHL elevada, acima de 400U/L (Tabela 6).

TABELA 6

Resultado da análise multivariada da associação de fatores clínico-laboratoriais associados ao óbito de pacientes portadores de HIV e fungos causadores de infecção sistêmica, em acompanhamento nas unidades especializadas para assistência ao portador de HIV/Aids, Mato Grosso, 2005-2008.

\begin{tabular}{|c|c|c|c|c|c|c|c|c|}
\hline \multirow[b]{3}{*}{ Fatores } & \multicolumn{4}{|c|}{ Óbito } & & & \multirow[b]{3}{*}{ IC95\% } & \multirow[b]{3}{*}{$\mathrm{p}$} \\
\hline & \multicolumn{2}{|c|}{$\operatorname{sim}$} & \multicolumn{2}{|c|}{ não } & \multicolumn{2}{|c|}{$\mathrm{OR}$} & & \\
\hline & $\mathrm{n}^{0}$ & $\overline{\%}$ & $\mathrm{n}^{0}$ & $\%$ & bruto & $\overline{\text { ajustado }}$ & & \\
\hline Etilismo & 20 & 54,1 & 17 & 45,9 & 8,2 & 10,8 & $1,12-104,12$ & 0,04 \\
\hline Idade (média) & 36 & 7,7 & 36 & 11,2 & 1,0 & 0,98 & $0,88-1,09$ & 0,76 \\
\hline Anemia & 18 & 56,2 & 14 & 43,8 & 4,4 & 2,96 & $0,44-20,11$ & 0,44 \\
\hline $\mathrm{DHL}>400 \mathrm{U} / \mathrm{L}$ & 21 & 61,8 & 13 & 38,2 & 13,7 & 20,7 & $1,42-303,59$ & 0,02 \\
\hline Linfócitos T CD4+ (média) & 90 & 97,7 & 82 & 79,8 & 0,9 & 0,99 & $0,98-1,00$ & 0,55 \\
\hline Albumina $<3 \mathrm{~g} / \mathrm{dL}$ & 13 & 72,2 & 5 & 27,8 & 3,4 & 1,68 & $0,28-10,18$ & 0,57 \\
\hline AST > $40(\mathrm{U} / \mathrm{L})$ & 16 & 42,1 & 22 & 57,9 & 0,9 & 0,33 & $0,03-3,57$ & 0,36 \\
\hline Uréia > $50 \mathrm{mg} / \mathrm{dL}$ & 13 & 65,0 & 7 & 35,0 & 4,4 & 1,25 & $0,20-7,57$ & 0,80 \\
\hline Pneumocistose associada & 10 & 58,8 & 7 & 41.2 & 2,74 & 2,67 & $0,31-22,6$ & 0,36 \\
\hline
\end{tabular}

OR: odds ratio, DHL: desidrogenase lática, AST: aspartato-aminotransferase, DP: desvio padrão.Obs: 51 pacientes foram analisados no modelo. 


\section{DISCUSSÃO}

No presente estudo, a prevalência (IC95\%) encontrada de micose sistêmica entre pacientes com HIV/Aids acompanhados nas unidades especializadas, para assistência ao portador de HIV/Aids de Cuiabá, Mato Grosso, no período de 2005-2008, foi de 4,6\% (3,6\%-5,9\%), excluindo-se o diagnóstico de pneumocistose, causada por Pneumocystis jiroveci, recentemente classificado como fungo ${ }^{39}$. Essa exclusão foi necessária, em virtude da dificuldade de confirmação diagnóstica da pneumocistose em nosso meio.

Em geral, foram pacientes que procuraram o serviço de saúde com mais de 30 dias de sintomas, adultos jovens e com maior proporção do sexo masculino, provavelmente, explicada pela ainda maior proporção de Aids entre homens no Estado de Mato Grosso ${ }^{21}$, quando comparada à relatada para o Brasil, hoje com razão de sexo próxima de $1: 1^{121}{ }^{25}$. Destacou-se o baixo nível de escolaridade desses pacientes, o que certamente está relacionado à presença da doença nas camadas menos favorecidas da população. Esse perfil não é diferente da distribuição da Aids observada no Brasil, que vem mostrando progressiva tendência à pauperização da epidemia na ultima década ${ }^{121}$. Ainda como reflexo da pauperização da epidemia, encontrou-se alta proporção de pacientes com relato de etilismo e/ou tabagismo na amostra estudada, já descrita para a população brasileira de classe menos favorecida, economicamente ${ }^{1027}$.

A evolução letal nesse grupo de pacientes deve ser considerada elevada (40\%) e pode ter sido consequência de diversos fatores. Por exemplo, o relato de infecção fúngica no passado foi constatado para importante parcela dos pacientes, o que motivou uso prévio de antifúngico sistêmico e suas consequências. Essa pressão exercida pela droga sobre o fungo poderia explicar a ocorrência de quadros mais graves da micose sistêmica, como já demonstraram outros autores, que observaram pacientes com candidíase oral e criptococose resistente ao fluconazol ${ }^{2234}$. Além disso, no momento do diagnóstico da micose sistêmica dos pacientes do estudo, outras infecções oportunistas e potencialmente graves também foram diagnosticadas nesse grupo de pacientes, tais como a pneumocistose, a tuberculose e a neurotoxoplasmose. Jain e cols ${ }^{15}$ relataram que, apesar da queda progressiva do número de mortes devido à Aids na era pós-TARV-P, a pneumocistose ainda permanece como a principal causa de mortalidade. Por sua vez, Santo $\mathrm{cols}^{35}$, avaliando causas de morte relacionadas à tuberculose no sistema de informação de mortalidade de São Paulo, concluíram que menções ao termo tuberculose praticamente dobram o coeficiente de mortalidade proporcional por essa causa e são diretamente relacionadas à epidemia do HIV/Aids daquele estado.

Os principais sinais e sintomas apresentados pelos pacientes do estudo foram compatíveis com as micoses sistêmicas diagnosticadas e estão de acordo com os observados por outros autores $^{12240}$. Alguns dos sinais e sintomas observados no estudo reforçam a hipótese de outras infecções oportunistas em concomitância com a micose sistêmica, tais como as convulsões na neurotoxoplasmose/neurocriptococose e a tosse seca e dispnéia na pneumocistose. Lamentavelmente, a confirmação etiológica dessas infecções não pôde ser documentada neste estudo, impedindo a mensuração de seu impacto nos resultados observados.

Embora não se possa afastar a influência da tuberculose, pneumocistose e neurotoxoplasmose na evolução fatal dos pacientes deste estudo, o achado de alta (40\%) letalidade das micoses sistêmicas não é inconsistente com a literatura. Em Cuba, por exemplo, de 211 autópsias realizadas em pacientes que faleceram de Hiv/Aids, a causa mortis foi atribuída às infecções fúngicas em $44,1 \%$ deles ${ }^{13}$.

Os achados laboratoriais de anemia, hipoalbuminemia, insuficiência renal e elevação das enzimas hepáticas e DHL estão de acordo com os descritos por outros autores ${ }^{83642}$ que também descreveram quadro laboratorial semelhante e indicam fase avançada da imunossupressão, que predispõe à evolução mais agressiva da infecção. De fato, todos os pacientes do estudo encontravam-se em fase adiantada da doença, atestada pela baixa contagem de linfócitos T $\mathrm{CD} 4^{+}$.

Observou-se excelente performance das técnicas laboratoriais empregadas para a confirmação diagnóstica da micose sistêmica, principalmente do exame micológico direto e da cultura para fungos. Pappalardo ${ }^{29}$, avaliando 35 pacientes com Aids e criptococose, demonstrou alta (90\%) sensibilidade do exame direto. Sensibilidade de $100 \%$ dessa técnica também foi registrada por Goldani \& Sugar ${ }^{11}$, em revisão de 27 casos de paracoccidioidomicose na Aids. Aspecto curioso do presente estudo foi 0 achado de hemoculturas positivas em 8 (13,3\%) pacientes com micose sistêmica. Esse aspecto tem sido associado a pior prognóstico da criptococose $\mathrm{e}^{3138} \mathrm{e} \mathrm{histoplasmos} \mathrm{e}^{28}$. Também, para a paracoccidioidomicose, há relato de sepse grave seguida de morte, na qual o fungo foi isolado de hemocultura ${ }^{4}$.

Criptococose foi a micose sistêmica mais frequentemente identificada nos pacientes deste estudo e também a responsável pela maior proporção de óbitos. Esse achado já foi observado por diferentes autores que encontraram letalidade variando de 10\%$73 \%$ e atestam que essa micose sistêmica é a causa mais comum de meningite grave em pacientes com AIDS, principalmente em países em desenvolvimento $0^{261630}$.

Analisadas isoladamente, as características dos pacientes aqui estudados que se associaram ao óbito causado pela micose sistêmica foram o etilismo, a hipoalbuminemia, a insuficiência renal, a elevação da DHL e a carga viral do HIV. Seria de esperar uma associação inversa entre a contagem de linfócitos CD4+, demonstrativa do nível de imunodepressão do paciente, com a evolução fatal da micose. No entanto, essa associação não foi observada no estudo e pode ser explicada pela baixa contagem inicial e pequena variabilidade dessa contagem em toda a amostra de pacientes. Com exceção do hábito etílico, todas as variáveis laboratoriais associadas com o óbito são demonstrativas de fase avançada da doença no momento da admissão do paciente e, portanto, é esperado que se relacionem ao pior prognóstico da micose no paciente com HIV/Aids. De fato, após análise ajustada, apenas o nível sérico de DHL e o etilismo permaneceram independente associado ao óbito dos pacientes. 
Embora estudo recente não tenha demonstrado impacto do abuso de álcool na mortalidade por HIV/Aids ${ }^{41}$, é sabido que o uso de drogas ilícitas e de álcool é importante modificador prognóstico de pacientes com essa infecção ${ }^{203233}$. No entanto, é possível que a influência do alcoolismo na evolução fatal, aqui observada seja devida à associação com outras complicações do abuso do álcool no organismo, tais como esteato-hepatite alcoólica, cirrose hepática e câncer ${ }^{1020}$, as quais não foram abordadas no desenho deste estudo. Outra dificuldade encontrada para explicar essa associação foi a não utilização de recurso mais preciso para mensurar a variável etilismo entre os pacientes estudados, o que pode ter resultado em erro classificatório e tendenciosidade na análise. Dessa forma, é fundamental prosseguir na investigação dessa relação entre etilismo e prognóstico das micoses sistêmicas no paciente com HIV/Aids, utilizando metodologia e ferramentas diagnósticas mais robustas.

Achado relevante foi a evidente e independente associação entre o nível sérico aumentado de DHL e o óbito causado pela micose sistêmica nos pacientes estudados. Esta enzima é encontrada em todas as células dos vertebrados, ocupando importante posição no metabolismo celular fora da via glicolítica. Funciona como enzima de ligação entre o metabolismo de proteínas e carboidratos e serve como indicadora de condições fisiológicas ou estruturais alteradas, desempenhando um papel importante em condições de estresse, tais como a inflamação, a hipóxia e/ou choque prolongados ${ }^{1419}$. Outros autores também têm observado associação entre elevação da DHL e pior prognóstico de infecções fúngicas sistêmicas ${ }^{2} 1823$ 43. Possíveis explicações para a elevação da DHL em quadros infecciosos, micóticos ou não, associados à Aids são relacionadas à disfunção orgânica múltipla associada à disseminação do processo infeccioso $0^{23} \mathrm{e} / \mathrm{ou}$ à síndrome reativa hemofagocítica, que é consequente à ativação inapropriada de monócitos, presente nas infecções graves, principalmente em estágios avançados da Aids ${ }^{79}$. Baseado nessa já conhecida associação, tem sido aventado que o nível da DHL pode ser útil no diagnóstico diferencial entre pneumocistose e histoplasmose, uma vez que o nível dessa enzima é geralmente maior que 600U/L na histoplasmose, diferente do encontrado em outros pacientes com HIV/Aids e infiltrado pulmonar ${ }^{378}$.

Assim sendo, é plausível concluir que as infecções fúngicas sistêmicas representaram importante causa de infecção oportunista e morte entre os pacientes com HIV/Aids da região e que o etilismo e a elevação do nível sérico de DHL podem ser características clínico-laboratoriais preditoras de pior prognóstico e evolução letal dessas micoses.

\section{REFERÊNCIAS}

1. Bastos FI, Szwarcwald CL. AIDS e pauperização: principais conceitos e evidências empíricas. Caderno de Saúde Pública 16: 65-76, 2000.

2. Benfield TL, Larsen-Helweg J, Bang D, Junge J, Lundgren JD. Prognostic Markers of short-term mortality in AIDS-associated Pneumocystis carinii Pneumonia. CHEST 119: 844-851, 2000.

3. Butt AA, Michaels S, Kissinger P. the association of serum lactate dehydrogenase level with selected opportunistic infections and HIV progression. International Journal of Infectious Diseases 6: 178-181, 2002.
4. Caseiro MM, Etzel A, Soares MCB, Costa SOP. Septicemia caused by Paracoccidioides brasiliensis (LUTZ, 1908) as the cause of death of an AIDS patient from Santos, São Paulo State, Brazil - A nonedemic area. Revista do Instituto de Medicina Tropical de São Paulo 47: 209-211, 2005.

5. Chaisson RE, Gallant JE, Keruly JC, Moore RD. Impact of opportunistic disease on survival in patients with HIV infection. AIDS12: 29-33, 1998.

6. Clark TA, Hajjeh RA. Recent trends in the epidemiology of invasive mycoses. Current Opinion in Infectious Diseases 15: 569-574, 2002.

7. Corcoran GR, Hail A-A, Flanders CleD, Geimer J, Patterson T. Markedly elevated serum lactate dehydrogenase levels are a clue to the diagnois of Disseminated histoplasmosis in patients with AIDS. Clinical Infectious Diseases 24: 942-942, 1997.

8. Daher EF, Silva JR GB, Barros FAS, Takeda CFV, Mota RMS, Ferreira MT, Oliveira AS, Martins JC, Araújo SMHA, Gutiérrez-Andrianzén OA. Clinical and laboratory features of disseminated histoplasmosis in HIV patients form Brazil. Tropical Medicine and International Heath 12: 1108-1115, 2007.

9. Favara BE. Hemophagocytic Lymphohistiocytosis: a hemophagocytic syndrome. Seminars in Diagnostic Pathology 9: 63-74, 1992.

10. Galduróz JC, Caetano R. Epidemiologia do uso de álcool no Brasil. Revista Brasileira de Psiquiatria 26: 3-6, 2004.

11. Goldani LZ, Sugar AM. Paracoccidiodomycosis and AIDS: an overview. Clinical Infectious Diseases 21: 1275-1281, 1995.

12. Gutierrez ME, Canton A, Sosa N, Puga E, Talavera L,. Disseminated Histoplasmosis in Patients with Aids in Panama: a Review of 104 cases. Clinical Infectious Diseases 40: 1199- 1202, 2005

13. Hernández EA, Paz VC, Fernández-Terán MLP,. Micosis oportunistas invasivas en el sida. Un estudio de 211 autopsias. Revista Iberoamericana de Micología 15: 33-35, 1998.

14. Hsu YC, Chou TY, Chen CF, Wang D, Su CL, Hu RT. Rat liver ischemia/reperfusion induced proinflammatory mediator and antioxidant expressions analyzed by gene chips and real- time polymerase chain reactions.Transplantation Proceedings 40: 2156-2158, 2008.

15. Jain MK, Skiest DJ, Cloud JW, Jain CL, Burns D, Berggren RE. Changes in mortality related to human immudodeficiency virus infection: comparative analysis of inpatient death in 1995 and in 1999-2000. Clinical Infectious Diseases 36: 1030-1038, 2003.

16. Jarvis JN, Harrison TS. HIV-associated cryptococcal meningitis. AIDS 21: 21192129, 2007.

17. Kaplan JE, Hanson D, Dworkin MS, Frederick T, Bertolli J, Lindegren ML, Holmberg S, Jones JL. Epidemiology of Human Immunodeficiency VirusAssociated Opportunistic Infections in the United States in the Era of Highly Active Antiretroviral Therapy. Clinical Infectious Diseases 30: 5-14, 2000.

18. Koduri PR, Chundi V, DeMaria P, Mizock BA, Patel AR, Weinstein RA. Reactive Hemophagocytic Syndrome: A new presentation of disseminated histoplasmosis in patients with AIDS. Clinical Infectious Diseases 21: 1463-1465, 1995.

19. Kolev Y, Uetake H, Takagi Y, Sugihara K. Lactate dehydrogenase-5 (DHL-5) expression in human gastric câncer: association with hypoxia-inducible factor (HIF-1 alpha) pathway, angiogenic factors production and poor prognosis. Annals of Surgical Oncology 15: 2336-2344, 2008.

20. Lewden C, Salmon D, Morlat P, Bévilacqua S, Eric J, Bonnet F, Héripret L, Costagliola D, May T, Chêne G, Mortality 2000 study group. Causes of death among human immunodeficiency virus (HIV)-infected adults in the era of potent antiretroviral therapy: emerging role of hepatitis and cancers, persistent role of AIDS. International Journal of Epidemiology 34: 121-130, 2005.

21. Ministério da Saúde. Boletim Epidemiológico - AIDS e DST, ANO IV, $27^{\circ}$ a $52^{\circ}$ Semana Epidemiológica julho/dezembro de 2006 e $01^{\mathrm{a}}$ a $26^{\mathrm{a}}$ Semana Epidemiológica janeiro/julho de 2007.

22. Mitchell TG, Perfect JR. Cryptococcosis in the era of AIDS -100 years after the discovery of Cryptococcus neoformans. Clinical Microbiology Reviews 8: 515-548, 1995.

23. Montaner JS, Hawley PH, Ronco JJ, Russel JA, Quieffin J, Lawson LM, Schechter MT. Multisystemic organ faliure predicts mortality of ICU patients with acute respitarory faliure secondary to AIDS-related PCP. Chest 102: 1823-1828, 1992. 
24. Mora DJ, Santos CTB, Silva-Vergara ML. Disseminated histoplasmosis in acquired immudodeficiency syndrome patients in Uberaba, MG, Brazil. Mycosis 51: 136$140,2007$.

25. Morales AU, Barreda PZ. Vulnerabilidad al VIH en mujeres en riesgo social. Revista de Saúde Pública 24: 822-829, 2008.

26. Moreira TA, Ferreira MS, Ribas RM, Borges AS. Criptococose: estudo clínicoepidemiológico, laboratorial e das variedades do fungo em 96 pacientes. Revista da Sociedade Brasileira de Medicina Tropical 39: 255-258, 2006.

27. Nascimento EC, Justo JS. Vidas errantes e alcoolismo: uma questão social. Psicologia: Reflexão e Crítica 13: 529-538, 2000.

28. Oliveira FM, Fernandes SS, Severo CB, Guazzelli LS, Severo LC. Histoplasma capsulatum fungemia in the patients with acquired immudeficiency syndrome: detection by lysis-centrifugation blood-culturing technique. Revista do Instituto de Medicina Tropical de São Paulo 49: 135-138, 2007.

29. Pappalardo MCSM. Criptococose em AIDS: estudo clínico e microbiológico em 35 pacientes acompanhados no "Instituto de Infectologia Emílio Ribas", São Paulo, de 1995 a 1997. Tese de Mestrado, Instituto de Pesquisa da Secretaria de Estado da Saúde de São Paulo São Paulo, SP, 2002.

30. Pappalardo MCSM, Paschoal RC, Melhem MSC. AIDS-associated central nervous system cryptococcosis: a Brazilian case study. AIDS 21: 1971-1983, 2007.

31. Pasqualotto AC, Severo AB, Oliveira FM, Severo LC. Cryptococcemia. An analysis of 28 cases with emphasis on the clincial outcome and its etiologic agent. Revista Iberoamericana de Micología 21: 143-146, 2004.

32. Petry NM. Alcohol use in HIV patients: what we Don't Know may hurt us. International Journal of STD \& AIDS 10: 561-570, 1999.

33. Samet JH, Nicholas HJ, Meli, S, Freedberg KA, Palepu A. Alcohol consumption and antiretroviral adherence among HIV-Infected persons with alcohol problems. Neurological, behavioral and environmental relations to drinling. Alcoholism: clinical \& Experimental Research 28: 572-577, 2004.
34. Sant'Ana PL, Milan EP, Martinez R, Telles FQ, Fereira MS, Alcântara AP, Carvalho MT, Colombo AL. Multicenter Brazilian Study of Oral Candida Species Isolated from Aids Patients. Memórias do Instituto Oswaldo Cruz 97: 253-257, 2002.

35. Santo AH, Pinheiro CE, Jordani MS. Causas múltiplas de morte associado a tuberculose no Estado de São Paulo, 1998. Revista de Saúde Pública 37: 714-721, 2003.

36. Sharma SK, Kadhiravan T, Banga A, Goyal T, Bathia I, Saha PK. Spectrum of clinical disease in a series of 135 hospitalised HIV-infection patients from North India. BMC Infectious Diseases 4: 52, 2004.

37. Shen YZ, Qi TK, Ma JX, Jiang XY, Wang JR, Xu QN, Huang Q, Liu XN, Sun H Q, Lu HZ. Invasive fungal infections among in patients with acquired immune deficiency syndrome at a Chinese university hospital. Mycoses 50: 475-480, 2007.

38. Sivasangeetha k, Harish BN, Sujatha S, Parija SC, Dutta TK. Cryptococcal meningoencephalitis diagnosed by blood culture. Indian Journal of Medical Microbiology 25: 282-284, 2007.

39. Stringer JR, Beard CB, Miller RF, Wakefield. A new name (Pneumocystis jiroveci) for Pneumocystis from Humans. Emerging Infectious Diseases 8: 891- 896, 2002.

40. Wadhwa A, Kaur R, Agarwal SK, Jain S, Bhalla P. AIDS-related opportunistic mycoses seen in a tertiary care hospital in North India. Journal of Medical Microbiology 56: 1101-1106, 2007.

41. Walley AY, Cheng DM, Libman H, Nunes D, Horsburgh CR Jr, Saitz R, Samet J. Recent use, homelessness and increased short-term mortality in HIV-infected persons with alcohol problems. AIDS 22: 415-420, 2008.

42. Wheat LJ, Chetchotiaskd P, Willians B, Connolly P, Shutt K, Hajjeh R. Factors associated with severe manifestations of Histoplasmosis in AIDS. Clinical Infectious Diseases 30: 877-881, 2000.

43. Visnegarwala F, Graviss EA, Lacke CE, Dural AT, Johnson PC, Atmar RL, Hamill RJ. Acute Respiratory Failure Associated with Cryptococcosis in Patients with AIDS: Analysis of Predictive Factores. Clinical Infectous Diseases 27:12311237, 1998. 